Capítulo 6

\title{
Caminares y miradas de la comunicación y cultura desde la vertiente Mattelart
}

DOI: https://doi.org/10.16921/ciespal.23.26

Edizon León- Ecuador-CIESPAL

\begin{abstract}
Mientras más andaba, más caminaba Andaba con la cabeza y caminaba con el corazón

Abuelo Zenón

No se puede producir un tipo de trabajo, sin haber acumulado, previamente, una reflexión crítica. Toda teoría social nos lleva a un proyecto o a un modelo de organización social. (Armand Mattelart, 2010)
\end{abstract}

\section{Introducción}

El caminares mencionado desde el título de este artículo, no está relacionado con mostrar únicamente un recorrido, y menos pretende ser una secuencia temporal de las múltiples reflexiones en el campo de la 
comunicación y la cultura que se han venido dando, sino tiene que ver en los aprendizajes de ese caminar, tanto desde la teoría como de la praxis, tomando en cuenta principalmente los aportes de los Mattelart a favor de la construcción de un pensamiento crítico que busque transformar relaciones de poder estructurales que han pretendido naturalizar unas inequidades históricas.

Desde su llegada a América Latina, específicamente a Chille en el año XXXX, Armard Mattelart muestra un interés especial por el aprendizaje cultural y político en esta región. Para entonces el pensamiento y la corriente del funcionalismo era escuela que predominaba en los ámbitos académicos y en los ejercicios profesionales sobre todo del periodismo latinoamericano. Su primera vinculación de estudios en el Centro de Demografía de la Universidad Católica de Chile, en Santiago de Chile, para continuar más adelante en el Centro de Estudios de la Realidad Nacional (CEREN) será la semilla de su pensamiento crítico a partir de la deconstrucción de las políticas que se estaban desarrollando en cuanto al control de la natalidad, que no solo pasaba por una cuestión demográfica, sino por una cuestión política dirigida desde la política de los Estados Unidos para América Latina, bajo el justificativo de que la causa de la pobreza era la ausencia de un control natal de la población, sobre todo en las comunidades rurales, donde se concentraba los mayores niveles de pobreza.

Un segundo punto, es mirar a la vertiente de los Mattelart, no como una filiación directa a las obras de estos autores -Armand y Michéllesino desde un análisis crítico sobre las aportaciones y producciones teóricas dentro de sus contextos históricos-políticos y que en su mayoría han derivado de una praxis investigativa crítica desarrollando una transdisciplinaridad articuladas de igual manera a una diversidad de metodologías, que preconfiguraron una transmetodología. Por tanto, se busca un repensar crítico de la producción de estos autores para la construcción de pensamiento propio y orientación de investigaciones encaminadas a la transformación socio-cultural. 
Se vuelve pensamiento crítico la producción de los Mattelart, en tanto, cuestiona desde sus análisis genealógicos históricos porque no solo hacen evidentes relaciones de dominación y explotación, sino que lo cuestionan y buscan desestabilizarlos. No hay una linealidad empírica en esta construcción de pensamiento, por el contrario, surge de confrontaciones con teorías anteriores, deconstrucciones, diálogos, cuestionamientos y negociaciones epistemológicas, sumado a rigor conceptual y metodológico. De esta manera, la transdisciplarinaridad no es únicamente un encuentro "feliz" de varias disciplinas, es la articulación motivada y justificada epistémicamente, donde se van encajando diálogos interdisciplinares y con ellos nuevos sentidos que generen nuevas miradas analíticas, pero sin perder el sustrato teórico.

Para Mattelart, esos encuentros interdisciplinares deben ser realizados definiendo bien las posiciones de cada una de las colaboraciones, su interrelación y configuración; de otro modo provocarán un debilitamiento conceptual y reflexivo de la problemática en su conjunto (Maldonado, 2015, pg., 98 referenciando a Mattelart A. \& Mattleart M., 1987b, p. 224).

Con esto quiero llegar al punto que cuando se analiza la comunicación en el contexto de la transdisciplinaridad junto con la cultura, no es un encuentro apretado, sino que parte de la lógica de entender que la comunicación no puede ser reflexionada desde su teoría y mucho más desde su práctica sin entender la cultura, no sólo desde una perspectiva contextual, que está presente, sino en las construcciones de nuestros sentidos cuando se juntan en el análisis a la comunicación y la cultura. El problema epistemológico, no es buscar cuál campo de conocimiento es la que determina a la otra, sino cómo se generan representaciones, verdades, estrategias de dominación desde lo comunicativo en la cultura. 


\section{Miradas y reflexiones desde distintos aportes}

El circuito de la renovación, producción, circulación y consumo del conocimiento no se da por fuera de las condiciones materiales de los sujetos, y menos aún por fuera de los procesos de construcción de subjetividades individuales y colectivas, y de las construcciones de las identidades culturales. En tal sentido, nosotros como identidades culturales estamos constituidos por un lado, por unas condiciones materiales e históricas específicas -materialismo histórico desde la perspectiva marxista-, que al mismo tiempo se establecen desde la construcción de discursos y regímenes de verdad impuestos y legitimados desde las instituciones de producción de conocimientos, de esta manera los epistemes se convierten en regímenes de verdad desde la teoría del discurso de Foucault, por otro lado, desde los distintos campos de la comunicación en su multifunción de relacionamiento e interacción social. Todo esto es lo que nos constituye y construye como "seres sociales", "seres culturales" y "seres comunicacionales".

(...) siempre hay condiciones de la identidad que el sujeto no puede construir. Los hombres y las mujeres hacen la historia pero no en condiciones elegidas por ellos. Son producidos en parte por las historias que hacen. Somos siempre construidos en parte por los discursos las practicas que nos constituyen, de tal manera que no podemos encontrar dentro de nosotros mismos como individuos o sujetos o identidades individuales el punto desde donde se origina el discurso o la historia o la práctica. (cursivas del texto original). (Hall S., compiladores Restrepo, Walsh y Vich, 2013, p. 348).

Esto nos clarifica los procesos complejos de interacción de la interdisciplinaridad dimensionada en el campo de experiencia social, en la praxis social envuelta de las relaciones de poder, expresada en cada una de las 'disciplinas', pero al mismo tiempo en la lógica del inter y de lo trans disciplinario que potencia la evidencia de la constitución del poder y sobre todo de la operatividad desde las estrategias de ese poder hegemónico. 
Por decirlo de alguna manera, hay unos sistemas culturales universales -imperialismo cultural- ${ }^{-1}$ y local que son constitutivos de un sistema económico capitalista imperial y donde lo componen estructuras comunicacionales (materializadas en grandes corporaciones de información y comunicación, y ahora también de redes sociales), que no solo dan forma a este sistema, sino que ayudan enormemente a operativizar dicho sistema.

Se vuelve necesario hacer unas puntualizaciones que pueden generar caer en teorías como el funcionalismo desde la comunicación, como lo hace explícito los Mattelart, afirmando que desde la sociología del funcionalismo otorgaba a los medios de comunicación como nuevos instrumentos de la democracia moderna que podían regular la sociedad, restando su capacidad de trasmisión cultural y ocultando su carácter reproductor de las ideologías y valores del sistema:

La sociología funcionalista consideraba los medios de comunicación, nuevos instrumentos de la democracia moderna, como mecanismos decisivos de la regulación de la sociedad y, en este contexto, no podrá sino defender una teoría acorde con la reproducción de los valores del sistema social, del estado existente. Escuelas de pensamiento crítico va a reflexionar sobre las consecuencias del desarrollo de esto nuevos de producción y trasmisión cultural...Descritos y aceptados por el análisis funcional como mecanismos de ajuste, los medios de comunicación resultan sospechosos de violencia simbólica y son temidos como medios de poder y de dominación. (Mattelart A., Mattelart M, 1997, p. 53)

Alimentados de los aportes que venían haciendo filósofos de la escuela de Frankfur que se radicaron en Estados Unidos y que procedían de una línea del marxismo no ortodoxo y de la crítica al eco-

1 "El conjunto de procesos por los que una sociedad es introducida en el seno del sistema moderno mundial y la manera en que capa dirigente es llevada, por la fascinación, la presión, la fuerza o la corrupción, a moldear las instituciones sociales para que correspondan con los valores y las estructuras del centro dominantes del sistema o para hacerse su promotor" (Schiller, (1976, citado por Mattelart A., Mattelart M, 1997, p. 80). 
nomicismo y por otro lado de la corriente naciente de los Estudios Cultural de Birmingham en los años sesenta, de esta manera empiezan a alejarse de la mirada ortodoxa y tradicionalista de la cultura. ${ }^{2}$

De hecho, es Raymond Williams de la Escuela de Birgmingham, en su Análisis de la Cultura quien construye una mirada de cultura más concreta a partir de cuestionar una concepción de cultura abstracta que la define como: "una descripción de un modo de vida particular que expresa ciertos sentidos y valores no solo en el arte y el aprendizaje, sino en las instituciones y en la conducta cotidiana" (Williams citado por Hall, 2013, 563). Con este concepto, no sólo pluraliza la cultura con lo cual rompe con esa homogenización y apela a las formas de construcción de sentidos y valores de unas culturas particulares y el otro aporte está en que introduce la cotidianidad en la cultura.

Lo interesante es que esta producción de sentidos está ligada a la manera de relacionarse, es decir, en la forma de comunicar-se, y con ello, liga la idea de comunidad, Hall (2013, p. 565) en ese sentido está de acuerdo con Williams cuando plantea que, "La comunidad humana crece por el descubrimiento de significados comunes y vías de comunicación comunes...”

Armand y Michélle Mattelart en su texto Historia de las teorías de comunicación (1997), profundizan de manera crítica en rol de la cultura dentro de la geopolítica global, a partir de las industrias culturales y de donde se configura el término la 'cultura de masas' cuya caracterización está determinada por una estandarización en la producción no de cultura específicamente pero si de bienes culturales desde la lógica de

2 La Escuela de Birmingham creada en 1964 en Inglaterra principalmente por Richard Hoggart, Raymont Williams, Edward P. Thompson y Stuart Hall, todos ellos de la corriente del marxismo, entre sus aportes está el cuestionar el mecanicismos y la subyugación de la superestructura a la estructura, la cultura sometida la determinación social y sobre todo económica. R. Williams desde la historia debate el cuestionamiento de la singularidad de la cultura y plantea que las culturas en plural, tomando referencia del materialismo dialéctico plantea que la historia está hecha de luchas, tensiones y conflictos entre culturas y modos de vida, y por supuesto, ligado a las formaciones de clase, sumando los aportes del intelectual italiano Gramci, en cuanto, al mecanicismo de ligar lo cultural a lo económico y con ello a la categoría de clase. Sumado a esto a una propuesta epistémica y metodológica de "hacer" historia desde abajo. 
serialización, estandarización y división del trabajo, encaminadas fundamentalmente al consumo masivo. ${ }^{3}$

La industria cultural fija de manera ejemplar la quiebra de la cultura, su caída en la mercancía. La transformación del acto cultural en un valor destruye su capacidad crítica y disuelve en él las huellas de una experiencia auténtica. La producción industrial sella la degradación de la función filosófica-existencial de la cultura. (Mattelart A., Mattelart M, 1997, p. 54.)

Si bien los Mattelart reconocen los aportes de Adorno y Horkheimer de mirar en estas industrias culturales la relación entre arte y tecnificación como productos-mercancías derivados del capitalismo, hacen notar la limitación frente al arte y la cultura, comprenden que en estos análisis se pierde de vista el carácter transformador, crítico y cuestionador que puede tener y debe tener el arte y la cultura, esto significa reivindicar el carácter político que tiene la cultura como productor de sentidos y significados que en el caso particular surgen de un contextos y continuidad de resistencia, la cultura como una experiencia única y propia.

Bien se podría decir que la cultura- incluso la cultura de masas- no debe jugarse desde la estigmatización y tampoco de la sacralización, y menos en esa transición entre la instrumentalización de las cosas a la instrumentalización de los individuos -la racionalidad técnica-, desactivando todo ese potencial insurgente y de resistencia en la construcción tanto de subjetividades como de identidades.

A propósito de lo político de la cultura, Bolívar Echeverría en el prólogo que hace a una traducción de un escrito de Walter Benjamín, quien también formó parte de la escuela de Frankfurt, hace un planteamiento

3 Adorno y Horkheimer en los años 40s en los Estados Unidos, crean el concepto de industria cultural, donde analizan la producción industrial de los bienes culturales como movimiento global de producción de cultura como mercancías. Donde la lógica utilitaria de los bienes culturales como películas, programas radiofónicos, revistas es similar al mismo esquema de organización y planificación del management que la fabricación de autos en serie. La producción de bienes estandarizados efectivos para el consumo. MM 1997. 
interesante que surge de la obra de este filósofo, en cuanto a la ampliación del campo de lo político:

...es la obra de un militante, de aquel que había reunido ser a lo largo de su vida, convencido de que, en la dimensión discursiva, lo político de juega -y de manera a veces decisiva- en escenarios aparentemente ajenos al de la política propiamente dicha. (Echeverría, B., 2003)

Lo que se rescata de estos análisis del campo de la cultura, es mirar las corrientes críticas de las que se nutren los Mattelart, de todas estas teorías a partir de cuestionarlas y muestran ese camino de la construcción de pensamiento crítico que se conjugará en la inter-transdiciplinaridad articulando varias disciplinas para profundizar sus aportes en el campo de la comunicación.

Podría decir que todas estas reflexiones -y obviamente muchas más- que los Mattelart desarrollan, les permite enlazar la comunicación y la cultura desde la economía política de la comunicación. La misma que toma de una manera crítica del circuito de la producción de bienes materiales, producción-circulación/consumo-reproducción, donde se suma la genealogía de una geopolítica de la comunicación, que Mattelart tomando de la obra de Foucault plantea una "arqueología de saberes de la comunicación" (1997), de esta manera construyen unas genealogías de las visiones geopolíticas de la comunicación, ampliando sistemas de comunicación, que no están únicamente ligados a los mass medias, medios de comunicación, al igual que las industrias culturales se establecieron industrias de las comunicaciones. Y por otro lado, configura como estos sistemas de comunicación -redes de comunicación- ha sido parte fundamental en la configuración de una hegemonía global del poder, como ejemplo estarían los imperios europeos que se consolidan en el siglo XIX.

Esta geopolítica de la comunicación en su libro Historia de la Comunicación, toma la referencia del concepto de 'economía-mundo' de Fernand Braudel y de 'sistema-mundo' de Inmanuel Walleinstein 
quienes marcan una globalidad que se configura en la lógica de la dominación logrando una hegemonía mundial, que da lugar a la creación de centros, zonas intermedias y periferias, y será esta lógica que permite sostener esta hegemonía, es un mapa de la desigual histórica del mundo. "La nueva visión del espacio mundial conduce a renovar el estudio de las relaciones internacionales en materia de cultura y comunicación" (Mattelart A., Mattelart M, 1997, p. 79).

De esta manera desarrollan lo que denominan la comunicación-mundo que da cuenta de tanto de la hegemonía de una centralidad de los países denominados del "primer mundo" determinada por un monopolio y control de los sistemas de comunicación siempre ligado al monopolio económico, y las periferias -de la comunicación- que lejos de tener un algún control de las comunicaciones terminan siendo "receptores-consumidores" de ese capital informativo proveniente del primer mundo.

La llamada mundialización como parte de la expansión del capitalismo global, y que ha encontrado en el desarrollo tecnológico ligado a las comunicaciones debe ser interpelado en su trasfondo político y no solamente descriptivo y hasta justificado y que ha ido generando estas dislocaciones y también disrupciones en su afán de estandarización-homogeneización en la misma concepción espacial, como bien lo plantea el intelectual caribeño Édouard Glissant:

La mundialización, concebida como un no-ha-lugar, podría conducirnos, en efecto, a una dilución estandarizada. Pero, para todos y cada uno de nosotros, en esa huella que va desde su lugar en el mundo, y vuelve, y añade otra ida y vuelta, consiste la única permanencia. El mundo, en su consumada totalidad, no puede considerarse razón suficiente, generalidad que traiga al mundo su propia generalización. La trama del mundo gana color con todas las particularidades, cuantificadas; con todos los lugares reconocidos. La totalidad no es eso que se ha dado en considerar el ámbito de lo universal. Es la cantidad infinita y ya ejecutada del infinito detalle de lo real. Y que, por ser al detalle, no es totalitaria (2006, p. 192). 


\section{Economía política de la comunicación}

El resultado de esa geopolítica de la comunicación, denominada de manera engañosa como globalización de la información o de la comunicación ligada a la globalización del capitalismo ha generado brechas de desigualdad irreconciliables, permite que se empiece a desarrollar a partir de los años sesenta la 'economía política de la comunicación', "Primero adoptan la forma de una reflexión sobre el desequilibrio (desigualdad) de los flujos de información y de productos culturales entre los países situados a uno y otro lado de la línea de demarcación del "desarrollo"” (Mattelart A., Mattelart M, 1997, p. 77). Pocos años después ya se hablaba de industrias culturales en un proceso de masificación y con la falsa promesa de una democratización de la cultura incentivada desde lo público gubernamental.

Entre los aportes de que hacen los Mattelart en su mayoría de obras, es ir desmontando desde sus método genealógico, teorías, términos, neologismos y conceptos que en algún momento y espacio de la historia, estaban asumidos como verdades, así por ejemplo, el concepto de "sociedad de masas" que hace parte de la cultura de masas, como dicen ellos, había sido una referencia fundamental para entender la naturaleza de la modernidad de los medios de comunicación, y si bien, no desmerecen la importancia de estas afirmación, lo que plantean es que a esta reducción o "exclusividad" como la denominan, pierde ese privilegio, en tanto, se complejiza con nuevos cuestionamientos y miradas tanto de la cultura y la sociedad como de las mismas comunicaciones, generando desplazamientos epistémicos reflejadas en nuevas significaciones como una concepción mas totalizadora se abordar la comunicación y cuyos sentidos radican en su articulación y no en cada una de sus partes que la componen y que a la final terminan si produciendo cambios en la estructuración de las sociedades.

La economía política de la comunicación tiene a las industrias culturales (libro, discos, cine, televisión, prensa y otros más) como uno de los elementos centrales de sus análisis en sus primeros inicios. Mas allá 
de las discusiones teóricas que analizan críticamente los Mattelart, hay una asociación entre el capitalismo y los 'productos culturales' y sobre la penetración en cada una de ellas como parte de la última fase del capitalismo de la globalización capitalista, donde muchos intelectuales miraban una destrucción e incluso una desaparición de las diferentes culturas localizadas, a través de sus estrategias de homogeización para volverlas funcionales al consumo y así alimentar y fortalecer al sistema.

Dado que no ocurrió esto al menos de manera generalizada, no se puede negar que han existido consecuencias, lo que Stuart Hall llama dislocaciones:

Sin embargo, esta última fase de la globalización capitalista, con sus compresiones y reordenamientos brutales a través del tiempo y del espacio, no han resultado necesariamente en la destrucción de aquellas estructuras específicas ni de los vínculos e identificaciones particulares que suponen las comunidades más localizadas a las que una modernidad homogenizante supuestamente reemplazaría. Naturalmente, las fuerzas de la modernidad capitalista, en sus desarrollos conjuntos y complejos, han dislocado radicalmente las sociedades a las que han penetrado... (Hall, 2013, p. 566).

Este autor también hace evidente que esas identidades surgidas o reafirmadas en las diversas identidades diferencias, sirvieron de "inspiración comercial" para producir bienes culturales en la misma lógica de industria cultural en un proceso de vaciamiento simbólico y de sentidos interpeladores de un sistema colonial-patriarcal-adultocétrico-heterosexual, por tanto, resultó un doble negocio, en el sentido de neutralizar de alguna forma ese agenciamiento político y por otro un buen negocio. "Sin embargo, la llamada "lógica del capital" ha operado a través de la diferencia -preservando y transformando la diferencia (incluida la diferencia sexual)- y no a través de socavarla”. (Ídem, p. 566), produciéndose otras formas de violencia simbólica y de apropiación cultural.

En la misma reflexión de Hall, los Mattelart coinciden en el sentido de la imposibilidad de la 'globalización cultural' de desaparecer 
las identidades locales y lo nacional llevando a esferas supranacionales regidas únicamente por leyes del mercado, y aprovechan para de paso para criticar al antropólogo Appadurai:

La neutralización del contexto estructural, en el que tiene lugar la producción y circulación de los flujos, armoniza con la tesis de los postnacional y su corolario, que s la caducidad del Estado-nación en un mundo desterritorializado...El axioma de [Apaddurai] es que el Estado-nación se ve desbordado por la multiplicidad de los flujos transfronteras (migratorios, mediáticos, técnicos, financieros, turísticos, etc.) y por la diversificación de las identidades y de las modernidades. (Mattelart, A., 2010, p. 216-7).

Más allá de la estrategia o de la lógica del capitalismo de apropiar y vaciar en la fabricación de bienes 'universales' globales de la diferencia o diversidad cultura, que ha dislocado pero no eliminado, se debe a un agenciamiento de éstas desde las diversas formas de resistir. Con esto, cuando se plantea temas como imperialismo cultural, globalización cultural, teorizan desde las acciones del capitalismo imperial y no desde el otro lado de la historia, desde esas periferias que están reinventándose desde sus tradiciones, mitos, espiritualidades, y procesos de apropiación estratégicos, etc. También se debe tomar en cuenta que los usos estratégicos y políticos de formas de comunicación para confrontar ese imperialismo cultural y comunicacional.

Pero obviamente no todo pasa por el conflicto y la tensión, también hay procesos de negociación cultural y comunicacional (en tanto formas comunicacionales), uno de esas negociaciones ha sido lo que han denominado la glocalización para explicar la interrelación de lo global con lo local, y algunos han ido mucho más allá al afirmar que en lo global o lleva lo local, y viceversa, dentro de este repertorio de negociaciones está lo que los antropólogos han trabajo como la hibridación cultural. En estas discusiones, Mattelart a más de criticar el neologismo de glocalización, argumentando que el término viene del marketing anglosajón Mattelart, y la hibridación por ser más de orden ideológica, se adscribe a la propuesta que hace Glissant en el concepto 
de creolización, en cuanto a los procesos y estrategias mediante el cual se ponen en contacto, tomando en cuenta, que muchos de estos contactos, no están dado desde consensos sino como imposiciones, "Pero la criollización es un mestizaje con un valor añadido, el que le confiere la imprevisibilidad" (Glisant, 2002, p. 21). Habría que añadir a esta última discusión la trampa de analizar epistémicamente desde la oposición binarista que es una herencia de la Ilustración.

\section{A manera de conclusión}

El trabajar reflexiones y aportes desde la vertiente Mattelart, significa no únicamente analizar la obra de los Mattelart, sino de la figura de Armand Mattelart sin duda en el contexto de su práctica política e intelectual, expresada a través de su gran y vasta producción. ${ }^{4}$ En tal sentido, pienso que hasta cierto punto no se ha hecho "justicia" con esa magna producción -cuantitativa y cualitativamente- en cuanto a la difusión y permanencia de la contemporaneidad de sus producción teórica, tanto a nivel de los intelectuales e investigadores que trabajan la comunicación, como tampoco de la introducción de sus obras dentro de los programas de estudios de las facultades comunicación.

Es por ello, que desde el Centro Internacional de Estudios para América Latina-CIESPAL y de manera específica la Cátedra Mattelart Economía y Políticas de Comunicaciones, que se creó en esa misma institución, está siendo fundamental para tanto la difusión como para aportar desde esta vertiente a la investigación y construcción de pensamiento crítico en la comunicación para toda la región de América Latina.

Muchas de las obras escritas por los Mattelart cuentan con algunas décadas desde sus publicaciones, más allá de estar descontextualizadas teóricamente con estos tiempos vertiginosos, su obra tiene

4 A lo largo de su muy fecunda obra teórica, histórica, política y crítica, Armand Mattelart produce más de 32 libros como autor y coautor y cientos de artículos especializados sobre los problemas centrales de la comunicación en América Latina, Europa y en el mundo en general. 
vigencia impresionante en los actuales momentos, lo cual muestra a más de la agudeza intelectual e investigativa, un sentido visionario de las configuraciones y el papel que jugarían los sistemas de comunicación e información en los actuales momentos.

Para entender los procesos de cultura y de comunicación en América Latina desde los Mattelart como se ha explicado en la argumentación de este artículo fue lo que les permitió pensar las bases de la economía-política para comprender la reproducción de dichos fenómenos en las sociedades contemporáneas y algunas vías para su transformación.

Todas estas reflexiones conducen inevitablemente a la conclusión de que sin del desarrollo de los sistemas de comunicación hasta nuestros días -en toda la dimensión genealógica y política que han trabajado los Mattelart -, incluyendo los sistemas de vigilancia desde las comunicaciones, no hubiera sido posible el desarrollo y quien sabe si la existencia del capitalismo, la globalización, la mundialización, el imperio cultural, la cultura y comunicación de masas, etc., ahí radica la importancia del pensamiento crítico comunicacionales que estos autores aportan, se trata en esta relación de cultura y comunicación articulada desde la economía política el desentrañar las formas y el contenido ideológico de una de las manifestaciones superestructurales más características de la sociedad de masas

La dificultad reside en que las potencias opresoras, que son multinacionales y están interesadas en llevar a cabo sus totalidad-tierra, donde pueden meterse en todo para sacar adelante con mal sus provechos, las ciudades mayores, el mínimo islote, recurren a una estrategia que aparenta ser mundialista. "!Abríos! No os encerréis en vuestra identidad". Y eso, en este caso, quiere decir: "Consentid en la imparable necesidad del mercado". Y, de esta forma, tienen la esperanza de diluirnos en el aire del tiempo. Algunos pueblos resisten. Sí, con dificultad. Porque la necesaria oposición puede a veces engendrar un enclaustramiento y, con una terrible agonía, ratificar la implícita amenaza que decreta el capitalismo. (Glissant, 2006, p. 192) 


\section{Referencias}

Echeverría. B. 2003. "Introducción”. En: Benjamin, La obra de arte en la época de reproductibilidad técnica. México D.F. ITACA

Hall, S. 2013. Sin garantías. Trayectorias y problemáticas en los estudios culturales. Restrepo. E., Walsh, C., y Vich, V. (compiladores). Quito. Corporación Editora Nacional.

Glissant, E. 2006. Tratado del Todo-mundo. Barcelona-España. Ediciones del Bronce.

2002. Introducción a una poética de lo diverso. Barcelona-España. Ediciones del Bronce.

Maldonado, E. 2015. Epistemología de la Comunicación. Análisis de la vertiente Mattelart en América Latina. Quito. Ediciones CIESPAL.

Mattelart, A. 2010. Por una mirada-mundo. Conversaciones con Michel Sénécal. Temuco-Chile. Universidad de la Frontera.

Mattelar, A., y Mattelart, M. 1997. Historias de las Teorías de Comunicación. España. PAIDOS. 

Capítulo 7.

\section{A supressão da voz feminina nos estudos comunicativo- midiáticos da América Latina. A ideia introdutória de 'mães' fundadoras do Pensamento Comunicacional'}

Maria Cristina Gobbi

Bolsista de produtividade do CNPq, Professora Livre docente da Universidade Estadual Paulista Júlio de Mesquita Filho (UNESP).

\section{Breve cenário e alguns de seus contextos}

Com uma cultura diversificada, uma geografia variada e um extenso território, a América Latina reúne 20 países com histórias que “corrieron paralelas y estuvieron unidas por semejanzas, pero también estuvieron marcadas por diferencias políticas, sociales e culturales" (Cano \& Barrancos, 2006, p. 547).

Neste contexto, o surgimento de espaços de reflexão e de pesquisa vem ao encontro dos processos de institucionalização que se desenvolveram em toda a região. Como afiança Corner (2019, p. 1), o “exame de como os programas de ensino e a atividade de pesquisa contribuíram para institucionalizar a área com uma identidade acadêmica discreta, embora muito debatida", também reforça o argumento. Estes estudos têm instigado a comunidade acadêmica para continuidade das investigações, buscando romper com a 'persistência da teoria negada', denunciada por Jesús Martin-Barbero, na década de 1982. (Fuentes Navarro, 2019) 